\title{
Circuit
}

Musiques contemporaines

\section{La contribution du Quatuor Molinari au rayonnement par le disque de la musique de R. Murray Schafer, Jean Papineau-Couture et Petros Shoujounian} The Recordings of the Molinari Quartet and their Contribution to the Musical Outreach of Composers R. Murray Schafer, Jean Papineau-Couture and Petros Shoujounian

\section{Alexis Raynault}

Volume 29, numéro 3, 2019

Bozzini, Molinari, Quasar : trio de quatuors

URI : https://id.erudit.org/iderudit/1066485ar

DOI : https://doi.org/10.7202/1066485ar

Aller au sommaire du numéro

Éditeur(s)

Circuit, musiques contemporaines

ISSN

1183-1693 (imprimé)

1488-9692 (numérique)

Découvrir la revue

Citer cet article

Raynault, A. (2019). La contribution du Quatuor Molinari au rayonnement par le disque de la musique de R. Murray Schafer, Jean Papineau-Couture et Petros Shoujounian. Circuit, 29(3), 45-53. https://doi.org/10.7202/1066485ar
Résumé de l'article

Interprète mondialement reconnu du répertoire contemporain et récipiendaire de nombreux prix, le Quatuor Molinari a enregistré près de quinze disques depuis sa fondation en 1997. Cet article traite de l'apport des disques du Quatuor Molinari au rayonnement de la musique de trois compositeurs canadiens - R. Murray Schafer, Jean Papineau-Couture et Petros Shoujounian - en le contextualisant historiquement par rapport à la genèse des oeuvres abordées et aux autres ensembles qui les ont jouées. 


\section{La contribution du Quatuor molinari Molinari au rayonnement par le disque de la musique de R. Murray Schafer, Jean Papineau- Couture et Petros Shoujounian}

Alexis Raynault

Le Quatuor Molinari, fondé en 1997 par la violoniste Olga Ranzenhofer, doit sa renommée tant à ses concerts marathons ${ }^{1}$ qu'à sa production discographique, soigneusement élaborée au cours des vingt dernières années.

Aux côtés de formations comme les quatuors Kronos, Arditti et Keller, le Molinari contribue au rayonnement des musiques de Zorn, Schnittke, Goubaïdoulina et Kurtág. Dans cette production, les quatuors à cordes du compositeur canadien R. Murray Schafer font, depuis 1999, l'objet d'une attention constante du groupe, tant sur disque qu'en concert. Fruit d'une collaboration étroite avec le compositeur, les quatuors $n^{\circ} 7, n^{\circ} 8, n^{\circ} 10, n^{\circ} 12$ et Alzheimer's Masterpiece, le $13^{\mathrm{e}}$, ont été écrits pour le Quatuor Molinari. Celui-ci a par ailleurs contribué à mieux faire connaître la musique de Jean Papineau-Couture en enregistrant, en 2016, l'intégrale de ses quatuors à cordes $^{2}$. La même année, l'ensemble enregistrait aussi les quatuors n ${ }^{\text {os }} 3$ à 6 du compositeur montréalais d'origine arménienne Petros Shoujounian.

Dans cet article, nous aborderons l'apport des disques du Quatuor Molinari au rayonnement de la musique de ces trois compositeurs canadiens, en le contextualisant historiquement par rapport à la genèse des œuvres abordées et aux autres ensembles qui les ont jouées.

\section{L'intégrale des quatuors de R. Murray Schafer}

Connu pour sa production universitaire ${ }^{3}$ et les monumentales œuvres de théâtre musical qui forment le cycle Patria, R. Murray Schafer laisse aussi
1. Au printemps 2015, par exemple, le quatuor a présenté en trois concerts l'intégrale des 15 quatuors de Chostakovitch.

2. Le disque est le fruit d'une collaboration du quatuor avec le Centre de musique canadienne au Québec.

3. Comme le World Soundscape Project. 
4. Cet événement a inspiré la parution d'un numéro de la présente revue intitulé Le quatuor à cordes selon Schafer (vol. 11, $\mathrm{n}^{\circ} 2,2000$ ).

5. Johanne Goyette, ancienne réalisatrice à la Société Radio-Canada, a fondé l'étiquette ATMA Classique en 1994.

6. Julie Trudeau remplace alors Sylvie Lambert au violoncelle.

7. Ces deux œuvres ont été créées par le Quatuor Orford.

8. Remplacé au printemps 2018 par Antoine Bareil. un important corpus de musique de chambre dominé par 13 quatuors à cordes, vaste parcours qui itère des idées importantes de son auteur, comme l'introduction de dimensions théâtrales et spatiales dans la performance et l'imitation, dans l'écriture, de phénomènes naturels.

Fondé en 1997, le Quatuor Molinari sera, dès ses débuts, l'ardent promoteur de l'œuvre pour quatuor à cordes de R. Murray Schafer, sollicitant le compositeur pour de nouveaux opus et reprenant les précédents en concert. La collaboration entre le groupe et le compositeur se cristallise autour d'un grand concert rétrospectif ${ }^{4}$ présenté à Montréal en décembre 1999, incluant la création du Quatuor $n^{\circ}$ 7. À la même époque, un album regroupant les quatuors $\mathrm{n}^{\text {os }} 1$ à 7 paraît chez ATMA Classique (2001). On y entend la première formation du Quatuor Molinari avec, entourant la fondatrice Olga Ranzenhofer, Johannes Jansonius (violon 2), David Quinn (alto) et Sylvie Lambert (violoncelle), tous trois membres de l'Orchestre symphonique de Montréal. Cette parution constitue également la première d'une longue suite de collaborations du groupe avec la réalisatrice et productrice Johanne Goyette ${ }^{5}$.

En 2003, toujours avec ATMA, le Quatuor Molinari ${ }^{6}$ enregistre le Quatuor $n^{\circ} 8$ - dont il est dédicataire - avec le monodrame Beauty and The Beast (1979) et le quintette avec harpe Theseus $(1983)^{7}$. Cet enregistrement du Quatuor $n^{\circ} 8$ se retrouve aussi sur le second volet du projet d'intégrale paru en 2013 et réunissant les quatuors $n^{\text {os }} 8$ à 12 . On entend sur ce dernier disque la seconde distribution du Quatuor Molinari, constitué d'Olga Ranzenhofer, Frédéric Bednarz ${ }^{8}$ (violon 2), Frédéric Lambert (alto) et Pierre-Alain Bouvrette (violoncelle). À ce jour, seul Alzheimer's Masterpiece, treizième et dernier opus du compositeur, n'a pas été porté sur disque.

R. Murray Schafer a eu le privilège de voir sa musique pour quatuor à cordes défendue très tôt - qui plus est, de manière pancanadienne - par des ensembles de premier plan déterminés à l'inscrire au canon du genre. Préparées avec soin et bénéficiant du rayonnement et de la valeur artistique et technique des productions d'ATma Classique, les interprétations de la musique de R. Murray Schafer par le Quatuor Molinari transcendent, par le disque, la niche exiguë de la création musicale savante contemporaine. Pour preuve, ces parutions ont reçu plusieurs récompenses, dont deux prix Opus du Disque de l'année - Musique actuelle, contemporaine, électroacoustique, d'abord pour les quatuors $n^{o s} 1$ à 7 (2001), puis pour le $8^{e}$ Quatuor, Theseus, Beauty and The Beast (2003); ce dernier disque a également reçu un Coup de Cœur de l'Académie Charles-Cros en 2004. Enfin, cette même année, R. Murray Schafer recevait le prix Juno de la Classical Composition of the Year pour le Quatuor no 8, commande du Molinari. 
Si les critiques recensées par aTma Classique sur son site soulignent l'éclectisme et la variété de l'inspiration de ce cycle de quatuors, le magazine Gramophone souligne quant à lui l'influence des grands cycles de Béla Bartók et Dmitri Chostakovitch sur les quatuors $n^{o s} 1$ à 7 , et compare l'apport de Murray Schafer au genre à celui de l'Américain Elliott Carter'.

Grâce à la commande d'œuvres nouvelles et à la défense des œuvres existant sur disque, la collaboration du Quatuor Molinari avec R. Murray Schafer aura contribué à faire connaître l'œuvre du compositeur à une audience internationale de mélomanes et à établir sa réputation comme figure incontournable de la musique classique contemporaine, notamment dans les cercles plus conservateurs de l'industrie du disque.

Deux autres formations de renom sont liées à l'élaboration du cycle des quatuors de R. Murray Schafer : les quatuors Purcell et Orford ${ }^{10}$. En résidence à l'Université Simon Fraser ${ }^{11}$, le Quatuor Purcell a créé les quatuors $n^{\circ} 1, n^{\circ} 2$ et $n^{\circ} 4$. Le Quatuor Orford a quant à lui assuré la création des quatuors $n^{\circ} 3$ et $n^{\circ} 5$ et enregistré, pour son $25^{\mathrm{e}}$ anniversaire (1990), l'intégralité des quatuors composés à ce jour, soit les quatuors $n^{\text {os }} 1$ à $5^{12}$. Lalbum, enregistré pour Centredisques $^{13}$ et récipiendaire d'un prix Juno, constitue un exemple rare pour son époque de monographie de musique canadienne réalisée par un seul ensemble. Cette réalisation est d'autant plus remarquable et significative qu'elle témoigne de l'engagement profond du Quatuor Orford, considéré un temps comme le premier quatuor du Canada ${ }^{14}$, en faveur de la création musicale canadienne.

\section{L'œuvre pour quatuor à cordes de Jean Papineau-Couture}

Si nous reconnaissons l'apport de Jean Papineau-Couture (1916-2000) à la vie musicale canadienne comme compositeur, administrateur et pédagogue ${ }^{15}$, ses œuvres, fruits de commandes passées par les grandes institutions culturelles et musicales du pays ${ }^{16}$, demeurent aujourd'hui bien peu souvent entendues hors du cadre scolaire et des concours musicaux ${ }^{17}$. Dans ces circonstances, la parution chez ATMA Classique de l'intégrale de ses quatuors à cordes constitue un événement significatif.

Jean Papineau-Couture apprend notamment son métier auprès de Nadia Boulanger, aux États-Unis, pendant la Seconde Guerre mondiale. Il subit d'abord l'influence de la musique française contemporaine et de celle de Stravinski, qu'il côtoie à cette époque. Partant du néoclassicisme, PapineauCouture abordera par la suite le total chromatique en s'inspirant des travaux théoriques de Paul Hindemith et en accordant une importance toujours plus grande aux dimensions du timbre et de la couleur, comme dans le trio Slanò.
9. s.a., s.d., "Schafer String Quartets Nos. 1-7".

10. Le Quatuor Purcell a été actif de 1968 à 1991 et le Quatuor Orford, de 1965 à 1991 .

11. R. Murray Schafer y a enseigné de 1965 à 1975 .

12. Le quatuor avait auparavant enregistré le Quatuor $n^{\circ} 2$, "Waves" en 1980 pour l'étiquette Melbourne (Mel SMLP-4038).

13. Étiquette fondée en 1981 par le Centre de musique canadienne pour faire la promotion du répertoire de concert canadien contemporain.

14. Il a bénéficié, à sa création (1965), du soutien de Gilles Lefebvre, fondateur des Jeunesses musicales du Canada et du Centre d'arts d'Orford.

15. Jean Papineau-Couture a contribué à la fondation du Centre de musique canadienne et de la Société de musique contemporaine du Québec. Il a également été doyen de la Faculté de musique de l'Université de Montréal de 1968 à 1973.

16. La Pièce concertante $n^{\circ} 5$, "Miroirs" (1963), par exemple, a été commandée par Zubin Mehta pour l'ouverture de la grande salle de la Place des Arts.

17. L'œuvre canadienne imposée du Concours international de musique de Montréal, édition 2016, était le premier mouvement de la Sonate en sol pour violon et piano. 
18. Clermont Pépin a écrit cinq quatuors à cordes et André Prévost, quatre. Le Quatuor Alcan a enregistré, pour la défunte Chaîne culturelle de Radio-Canada, les quatuors n ${ }^{\text {os }} 2$ à 4 de ce dernier.

19. Notamment celles de Jean Papineau-Couture, de Clermont Pépin, d'Otto Joachim et de John Weinzweig.

20. Violon solo de la Symphonie féminine de Montréal de 1940 à 1961

21. Otto Joachim (1910-2010), altiste, gambiste et compositeur de musique électronique et instrumentale. Le Quatuor Molinari a enregistré son Quatuor à cordes $n^{\circ} 2$ en 2000.

22. Cet enregistrement se retrouve sur le volume de l'Anthologie de la musique canadienne consacré à Jean Papineau-Couture, Radio-Canada International, ACM 4.

23. Dans l'esprit de filiation évoqué plus haut, soulignons que le Quatuor Morency avait également à son répertoire la musique de R. Murray schafer.

24. La composition de Slanò a été sollicitée par Gilles Lefebvre alors qu'il était directeur du Centre culturel canadien à Paris (1972-1978).

25. Accessible via MusiFlots, l'outil d'écoute en continu du Centre de
Avec ses cadets Clermont Pépin (1926-2006) et André Prévost (1934-2001), il est un des rares compositeurs québécois à avoir constitué un corpus suivi d'œuvres pour quatuor à cordes ${ }^{18}$. Témoignant de son évolution créatrice, ces œuvres, par leur genèse, mettent en lumière la filiation du Quatuor Molinari avec d'autres ensembles québécois et canadiens l'ayant précédé dans l'élaboration et la promotion d'un répertoire «national» d'œuvres pour quatuor à cordes.

Le Quatuor $n^{\circ}$ 1, composé en 1953 et créé la même année à Forest Hill (Toronto) par le Quatuor Spivak, appartient à la période néoclassique de la production du compositeur. Au Québec, l'œuvre a été jouée par le Quatuor à cordes de Montréal (actif de 1955 à 1963), premier ensemble professionnel à accéder à une notoriété dépassant les frontières. Pavant la voie au futur Quatuor Orford, le Quatuor à cordes de Montréal jouera beaucoup la musique des compositeurs canadiens ${ }^{19}$, aux côtés d'œuvres des répertoires classiques et modernes. L'ensemble aura sur la vie musicale canadienne une influence déterminante, notamment par l'activité pédagogique de Mildred Goodman ${ }^{20}$ (violon 2) à l'Université McGill et, au Conservatoire de musique de Montréal, des frères Otto ${ }^{21}$ (alto) et Walter Joachim (violoncelle). Ce dernier y aura pour élèves Marcel Saint-Cyr et Denis Brott, qui deviendront tous deux membres du Quatuor Orford. Le Quatuor ${ }^{\circ} 1$ de Jean PapineauCouture a aussi été enregistré en 1972 par le Quatuor classique de Montréal (actif de 1968 à 1976) pour Radio-Canada International22.

Le Quatuor nº 2, composé en 1967 pour le Quatuor Orford, a été enregistré en 1970 par ce même ensemble pour Radio-Canada International ; cet enregistrement a été réédité en disque compact en 2002 pour le second volume de la série Ovation. Quant au Quatuor nº 3, il a été créé en 1997 par le Quatuor Morency, un groupe constitué de musiciens liés à l'Orchestre Métropolitain et dont Olga Ranzenhofer a fait partie, à titre de second violon, de 1987 à la fondation du Quatuor Molinari dix ans plus tard ${ }^{23}$. Enfin, le Quatuor $n^{\circ} 4$, découvert en 2016 et resté inachevé, a été créé le 10 septembre de la même année par le Quatuor Molinari, à l'occasion d'un concert donné au Centre de musique canadienne au Québec pour le $100^{\mathrm{e}}$ anniversaire de naissance de Jean Papineau-Couture.

Le trio Slanò complète l'album²4. Écrite à l'intention du Trio Stradivarius, un ensemble mené par le réputé violoniste Gérard Poulet, l'œuvre requiert de ses interprètes une virtuosité consommée et déploie, dans des gestes violents et complexes, une grande variété de textures. De la création de l'œuvre, en 1976 , subsiste un enregistrement radiophonique ${ }^{25}$. Un autre enregistrement, celui-là réalisé en 1988 par les musiciens du Quatuor Accordes de 
Toronto, se retrouve sur l'anthologie d'œuvres de Papineau-Couture parue en 1988 chez Centredisques.

L'apport du Quatuor Molinari à la reconnaissance de la musique de Jean Papineau-Couture est inestimable, par sa haute tenue artistique et parce qu'il prend le contrepied d'une discographie autrement éparse et confidentielle. Pour la grande majorité produits et distribués par la Société Radio-Canada, les enregistrements disponibles des œuvres de Papineau-Couture ont subi les aléas inhérents à la dissolution progressive des différentes entreprises d'enregistrement de la Société à partir des années 1990.

Parmi les critiques du disque recensées par atma Classique sur son site, trois révèlent l'importance de la notoriété acquise par le Quatuor Molinari et l'étiquette dans la découverte de la musique de Jean Papineau-Couture hors des frontières canadiennes, contribuant à l'inscrire dans le contexte plus large de la création musicale européenne et américaine. Ainsi, dans le magazine Diapason, Pierre Rigaudière écrit que Papineau-Couture «compte parmi les nombreux compositeurs dont on ignorerait l'existence sur le Vieux Continent sans l'ambassade d'interprètes de renom ${ }^{26}$. » Sur le site MusicWeb International, Stephen Greenbank abonde dans le même sens :

I recently reviewed a $\mathrm{CD}$ from ATMA Classique of the complete string quartets by György Kurtág. They were given thrilling performances by the Canadian Quatuor Molinari. Having an inquisitive mind and always eager to delve into the music of less-well-known composers, I was eager to explore their new release of the complete string quartets of Jean Papineau-Couture, a name I've never come across before ${ }^{27}$.

Cette curiosité est également partagée par Dean Frey, du blogue Music for Several Instruments:

2016 is the centennial year for a number of composers, notably Alberto Ginastera and Milton Babbitt. I hadn't heard anything about celebrations for Jean Papineau-Couture, who was born in Montreal on November 12, 1916, until this new ATMA Classique disc came along. It's an important release from the Quatuor Molinari, who I know from their excellent Kurtág, Gubaidulina and Schnittke albums for ATMA $^{28}$.

\section{Petros Shoujounian : Noravank}

L'anthologie Noravank, parue en 1997 sous étiquette Société nouvelle d'enregistrement $(\mathrm{SNE})^{29}$, constitue le seul enregistrement commercial des compositions $^{30}$ de Petros Shoujounian. Comme l'album Klezmer Dreams de 2017, sur lequel figure une œuvre du compositeur et chef d'orchestre russo-canadien Airat Ichmouratov, il constitue un témoignage supplémentaire de l'intérêt du Quatuor Molinari pour les musiques d'Europe orientale ${ }^{31}$.
26. Rigaudière, 2017, p. 96.

27. Greenbank, s.d.

28. Frey, s.d.

29. Société nouvelle d'enregistrement (SNE), étiquette fondée en 1978 par Gilles Poirier, ancien réalisateur à la Société Radio-Canada.

30. Ses arrangements et orchestrations figurent toutefois sur les disques Hov Arek et Joyous Light, ce dernier mettant en vedette la soprano canadoarménienne Isabel Bayrakdarian.

31. Chez ATMA Classique, les quatuors à cordes de Schnittke, Gubaidulina et Kurtág. 


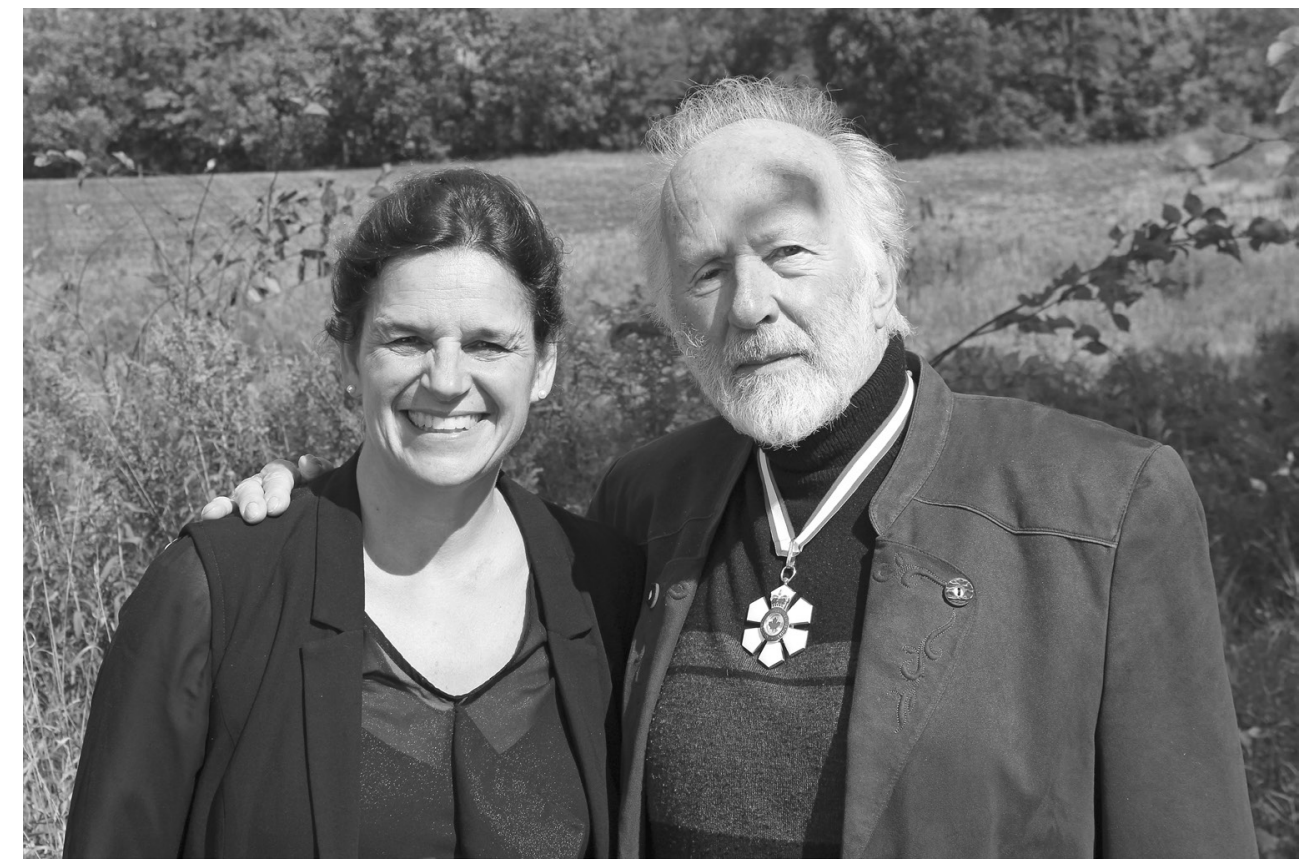

Olga Ranzenhofer et R. Murray Schafer, septembre 2014.

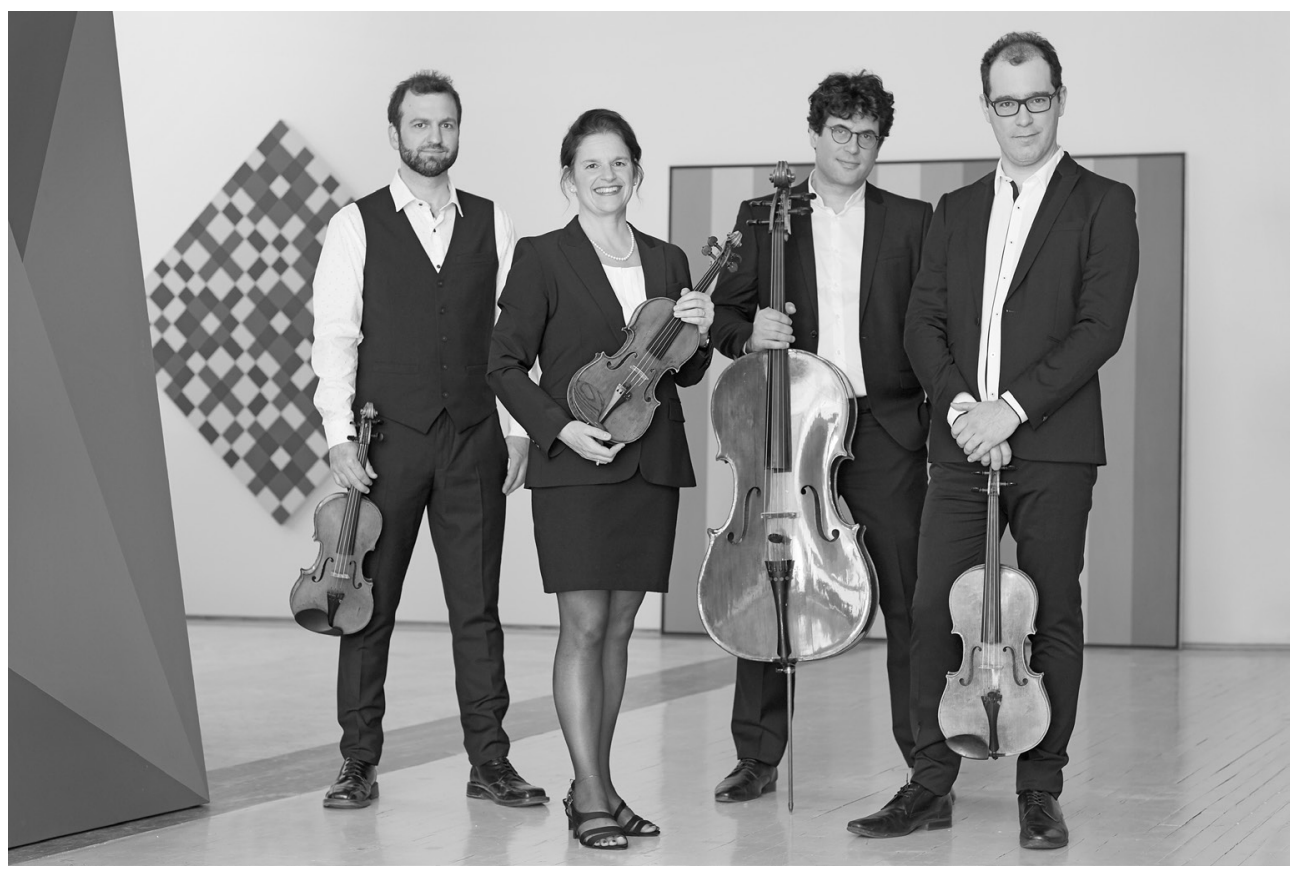

Le Quatuor Molinari, 2018. Crédit: Elizabeth Delage. 
Noravank, du nom d'un monastère arménien, est également le titre donné à l'album des quatuors à cordes (n ${ }^{\text {os }} 3$ à 6) de Petros Shoujounian, paru en 2016 chez atma Classique. Né en 1957 à Gyumri en Arménie et installé dès 1976 à Montréal, où il a fréquenté la classe de composition de Gilles Tremblay, Petros Shoujounian s'est impliqué dans la vie musicale de la diaspora ${ }^{32}$ ainsi qu'à l'Orchestre Métropolitain et au Festival Orford Musique. Comme celle de Tremblay, la musique de Shoujounian porte la marque d'une foi profonde, ce à quoi les œuvres enregistrées par le Quatuor Molinari ne font pas exception: les quatuors à cordes n ${ }^{\circ s} 3$ à 6 sont librement inspirés de chants liturgiques médiévaux transcrits par le musicologue Nikoghos Tahmizian. Chaque mouvement porte aussi le nom d'un cours d'eau d'Arménie, ancrant profondément ces œuvres dans le terroir.

Dépassant la seule mention de l'influence du chant liturgique arménien sur les compositions, certaines critiques recensées par ATMA inscrivent la musique de Petros Shoujounian dans le contexte plus large des courants esthétiques contemporains dominants. Ainsi, dans le magazine torontois The Whole Note, Vanessa Wells mentionne que l'écoute de Noravank lui a été suggérée par un amoureux de la musique d'Arvo Pärt, une parenté qu'elle dénote dans le Quatuor $n^{\circ} 3^{33}$. Dans The American Record Guide, Georges Adams situe quant à lui le Quatuor $n^{\circ} 6$ dans le sillage du minimalisme répétitif américain ${ }^{34}$.

Ici, la contribution du Quatuor Molinari aura été de porter à l'attention d'une large audience une production originale et unique en raison du métissage culturel qu'elle opère et de faire connaitre et apprécier cette spécificité propre à la scène montréalaise.

\section{Un outil de diffusion}

Aussi succincte soit-elle, la présente étude montre que le disque continue à ce jour de jouer un rôle important dans la diffusion et la connaissance de la musique canadienne, contribuant à faire découvrir le travail de ses créateurs et de ses interprètes au pays et à l'étranger. À cet égard, le succès du Quatuor Molinari s'explique tant par le sérieux et l'intérêt de sa démarche interprétative que par la solidité de l'étiquette aTMa Classique, son réseau et l'estime qu'on lui porte.

Dans une ère de transformation rapide des habitudes d'écoute et de consommation de la musique, de même que des manières d'archiver et de diffuser la connaissance, il sera intéressant d'observer comment les acteurs actuels du monde musical sauront tirer parti de ces transformations pour favoriser la découvrabilité des œuvres et des artistes. Il faudra encore trouver
32. Notamment comme chef du chœur Knar de l'Association culturelle arménienne Hamazkain de Montréal. Un album réalisé en 1984 témoigne de cette implication.

33. Wells, 2016.

34. Adams, Lamper et Sininger, 2017, p. 243. 
comment conjuguer l'accessibilité des plateformes d'écoute en continu avec la richesse documentaire et artistique - textes d'accompagnement et présentation visuelle étoffée - de l'objet disque.

\section{B I B L I O G RAP H I E}

Adams, Stephen J. (1982), R. Murray Schafer, Toronto, Toronto University Press.

Adams, George, Lamper, Kraig et Sininger, Richard (2017), «Newest Music », American Record Guide, vol. 80, n 1, p. 243.

Bail Milot, Louise (1986), Jean Papineau-Couture: la vie, la carrière et l'œuvre, LaSalle, Hurtubise.

Davoine, Françoise (1990), «L'aventure du disque de musique québécoise : bilan d'une décennie et perspectives d'avenir », Circuit, musiques contemporaines, vol. 1, nº 2, p. 81-92.

Elliot, Robert W. A. (1990), The String Quartet in Canada, thèse de doctorat, Université de Toronto.

Portugais, Jean et Ranzenhofer, Olga (2000), «Îles de la Nuit: parcours dans l'œuvre pour quatuor à cordes de R. Murray Schafer», Circuit, musiques contemporaines, vol. 11, $\mathrm{n}^{\circ} 2$, p. $15-54$.

Rigaudière, Pierre (2017), «Jean Papineau-Couture: les quatre quatuors à cordes. Slanò», Diapason, $\mathrm{n}^{\circ} 655$, mars, p. 96.

\section{ADRESSES URI}

FreY, Dean, s. d., "Quartets from an important Canadian modernist», Music for Several Instruments, https://several-instruments.blogspot.com/2016/10/quartets-from-importantcanadian.html (consulté le 21 juillet 2019).

Greenbank, Stephen, s. d., «Jean Papineau-Couture (1916-200o)», http://www.musicwebinternational.com/classrev/2017/Feb/PapineauC_quartets_ACD22751.htm (consulté le 21 juillet 2019).

Shoujounian, Petros, s. d., MusicaUnica, http://www.musicaunica.com/index.html (consulté le 21 juillet 2019).

Spier, Susan, Elliott, Robin et Edwards, Barry J., s. d., "Quatuor à cordes Orford/Orford String Quartet » in L'Encyclopédie canadienne, www.thecanadianencyclopedia.ca/fr/article/ quatuor-a-cordes-orfordorford-string-quartet (consulté le 21 juillet 2019).

Turbide, Nadia et Papineau-Couture, Isabelle, s. d., "Quatuor à cordes de Montréal/Montreal String Quartet» in L'Encyclopédie canadienne, www.thecanadianencyclopedia.ca/fr/article/ quatuor-a-cordes-de-montrealmontreal-string-quartet (consulté le 21 juillet 2019).

Wells, Vanessa (2016), «Noravank: Petros Shoujounian - String Quartets 3-6 - Quatuor Molinari », The WholeNote, https:/www.thewholenote.com/index.php/booksrecordsz/ moderncontempo/26074-noravank-petros-shoujounian-string-quartets-3-6-quatuor-molinari (consulté le 21 juillet 2019).

s. a., s. d., «Schafer String Quartets Nos. 1-7», Gramophone, https:/www.gramophone.co.uk/ review/schafer-string-quartets-nos-1-7 (consulté le 21 juillet 2019).

\section{ENREGISTREMENTS MUSICAUX}

Glick, Srul Irving, Golijov, Osvaldo, Ichmouratov, Airat, Kovács, Béla et Prokofiev, Sergei (2017), Klezmer Dreams, Quatuor Molinari, André Moisan (clarinette) et Jean Saulnier (piano), ATмa Classique, ACD22738. 
Papineau-Couture, Jean (2016), Quatuors à cordes nos 1-4, Trio Slanò, Quatuor Molinari, ATMA Classique, ACD22751.

Schafer, R. Murray (1990), Schafer: 5. Five String Quartets, Orford String Quartet et Rosemarie Landry (soprano), Centrediscs/Centredisques CD-CMCCD 39/4090.

Schafer, R. Murray (2000), String Quartets 1-7, Quatuor Molinari, ATMA Classique, ACD22188.

Schafer, R. Murray (2003), $8^{e}$ Quatuor, Theseus, Beauty and the Beast, Quatuor Molinari, Julie Nesrallah (mezzo-soprano), Jennifer Schwartz (harpe), ATMA Classique, ACD22201.

Schafer, R. Murray (2012), Quatuors à cordes 8-12, Quatuor Molinari, atma Classique, ACD22672.

Shoujounian, Petros (2016), Noravank. Quatuors à cordes nos 3-6, Quatuor Molinari, ATma Classique, ACD22737.

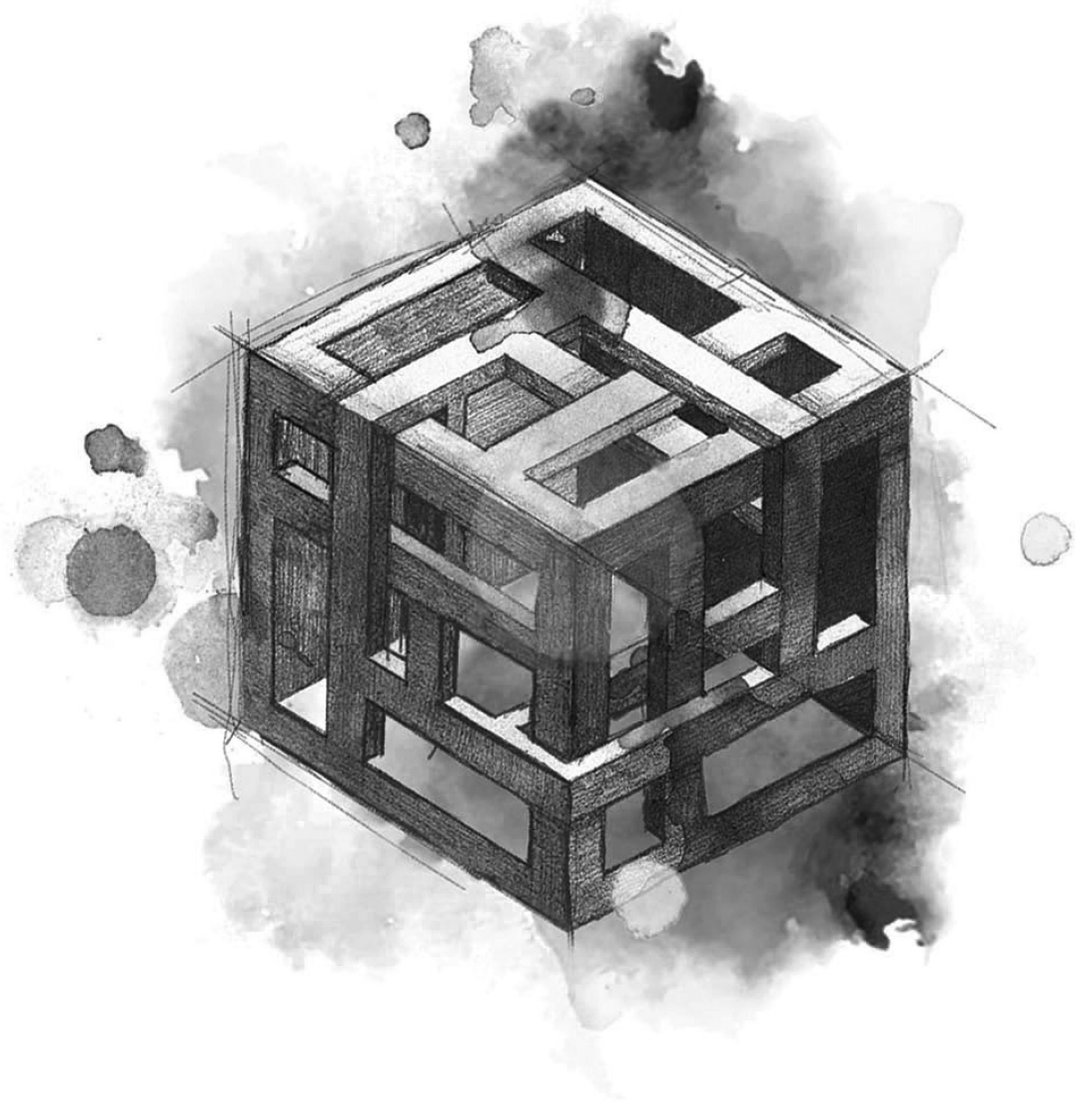

Camila Paz, Écho d'une cage, 2019. Aquarelle et crayon sur papier, $30 \times 30 \mathrm{~cm}$. 